INPLASY

PROTOCOL

To cite: Feng et al. Efficacy and Safety of abdominal

Acupuncture for InsomniaA protocol of systematic review and meta-analysis. Inplasy protocol 2021100088. doi: 10.37766/inplasy2021.10.0088

Received: 23 October 2021

Published: 23 October 2021

Corresponding author: Zhihong Wang

812839353@qq.com

Author Affiliation:

Changchun University of

Traditional Chinese Medicine

College of Acupuncture and

Tuina.

Support: Research

Development Program.

Review Stage at time of this submission: Preliminary searches.

Conflicts of interest:

None declared.

\section{Efficacy and Safety of abdominal Acupuncture for Insomnia A protocol of systematic review and meta-analysis}

Feng, ZT1; Wang, ZH²; Qiu, ZS3; Li, T4; Zhang, LL5; Wang, JJ6; Ying, DS7.

Review question / Objective: This systematic review protocol aims to provide the methods used to evaluate the effectiveness and safety of abdominal acupuncture therapy for treating insomnia.

Condition being studied: Insomnia is a subjective symptom of poor sleep quality and low sleep duration due to personal reasons such as inability to fall asleep or easy to wake up and difficulty in falling asleep, Acupuncture is a treatment that promotes health by inserting needles into specific acupoints on the human body at an appropriate depth. Acupuncture treatment of insomnia has the characteristics of simple operation, non-toxic side effects, positive curative effect, and high safety. Abdominal acupuncture is an unusual acupuncture therapy that treats systemic disorders by acupuncturing the viscera and meridians in the abdomen; Western medicine believes that it is the brain-gut axis, which is the theoretical basis of abdominal acupuncture for the treatment of insomnia. The brain-gut axis refers to a bidirectional regulatory system of interactions between the gastrointestinal tract and the brain.abdominal acupuncture stimulates the abdominal tract and improves sleep quality by regulating the secretion of sleep-related brain-gut peptide content through the brain-gut axis.

INPLASY registration number: This protocol was registered with the International Platform of Registered Systematic Review and Meta-Analysis Protocols (INPLASY) on 23 October 2021 and was last updated on 23 October 2021 (registration number INPLASY2021100088).

\section{INTRODUCTION}

Review question / Objective: This systematic review protocol aims to provide the methods used to evaluate the effectiveness and safety of abdominal acupuncture therapy for treating insomnia. 
Rationale: At present, Abdominal acupuncture is widely used in China for the treatment of insomnia. Abdominal acupuncture therapy is a special type of acupuncture therapy that treats systemic diseases by adjusting the viscera/ meridians (including innate and acquired meridians) through acupuncture at abdominal acupoints. According to Traditional Chinese Medicine, gastrointestinal disorders can lead to poor sleep quality. While the Western medicine believes that the brain-gut axis is the theoretical basis fro Abdominal acupuncture in the treatment of insomnia.

Condition being studied: Insomnia is a subjective symptom of poor sleep quality and low sleep duration due to personal reasons such as inability to fall asleep or easy to wake up and difficulty in falling asleep,Acupuncture is a treatment that promotes health by inserting needles into specific acupoints on the human body at an appropriate depth. Acupuncture treatment of insomnia has the characteristics of simple operation, nontoxic side effects, positive curative effect, and high safety. Abdominal acupuncture is an unusual acupuncture therapy that treats systemic disorders by acupuncturing the viscera and meridians in the abdomen; Western medicine believes that it is the brain-gut axis, which is the theoretical basis of abdominal acupuncture for the treatment of insomnia. The brain-gut axis refers to a bidirectional regulatory system of interactions between the gastrointestinal tract and the brain. Abdominal acupuncture stimulates the abdominal tract and improves sleep quality by regulating the secretion of sleep-related brain-gut peptide content through the brain-gut axis.

\section{METHODS}

Search strategy: The following databases will be searched from inception to October 2021: PubMed, Embase, CENTRAL, Web of Science, CNKI, VIP, CBM, Wan Fang Data, the search terms ("abdominal acupuncture") AND ("insomnia") AND "Random"" were used in the Englishlanguage databases, while shimian, bumei, fuzhen, and suiji were used in the Chineselanguage databases.

Participant or population: Participants a diagnosed with insomnia were included. Diagnosis criteria include the Diagnostic and Statistical Manual of Mental Disorders (DSM-V,DSM-5), International Classification of Sleep Disorders (ICSD-3), International Statistical Classification of Diseases and Health-Related Problems (ICD-10), and Chinese Classification of Mental Disorders (CCMD-3). No restrictions will be applied to age, gender, ethnicity, or source of cases. Participants with a diagnosis of insomnia will be included regardless of their age, gender, race, education or economic status.

Intervention: The experimental group was treated with abdominal acupuncture.

Comparator: The control group was treated with any therapies, such as Western medicine, acupuncture, moxibustion, auricular needle, and other conventional therapies. The following comparisons were made. 1. AA with Chinese herbal medicine; 2. AA compared with western medicine; 3. AA compared with placebo treatment; 4. AA compared with acupuncture alone; 5. AA compared with moxibustion alone. If the two groups received the same additional active therapy on the basis of the control treatment, the study can also be included.

Study designs to be included: Randomized controlled trials (RCTs) of abdominal acupuncture therapy for insomnia will be included. Non-RCTs, uncontrolled clinical trials, reviews, case reports, animal experiments, meta-analysis and repeated publications will be excluded.

Eligibility criteria: We will first use Note Express software (V.3.2) to remove duplicates, and then screen the retrieved studies separately by two reviewers (ZLL and QZS) according to the inclusion criteria. Two reviewers (ZLL and QZS) will exclude the papers that do not meet the inclusion criteria by reading the titles and abstracts. Then, the reviewers will check the full texts to determine the final decision 
according to the criteria. All the screening processes were conducted independently. If the articles information is insufficient, we will try to contact the authors to obtain the necessary details. When two reviewers have different opinions, the final decision will be made by a third reviewer (LT). The selection flow process is shown in the PRISMA flow chart.

Information sources: Two independent reviewers (QZS and WJJ) will search the following eight databases from the inception to October 2021; including CNKI, VIP database, CBM, Wan Fang Data Chinese Database, PubMed, CENTRAL, Web of Science, Embase. The combined method of MeSH term and free words was used for literature retrieval. There were no restrictions on language or publication status. The search strategy for PubMed is shown in Table 1. The search strategies of the other databases were established similarly. To augment the results of the database search, the bibliographies of the identified studies, relevant reports, and reviews will be manually searched. We will also contact the relevant experts and organizations for information about unpublished and ongoing studies.

Main outcome(s): Sleep quality will be evaluated using the Pittsburgh Sleep Quality Index (PSQI)as the primary outcome. Improvement in insomnia can be measured by the clinical efficacy and clinical cure rate. Outcomes can be measured simply at the end of treatment. Possible primary outcomes included the following: 1.Improvement in overall symptoms of insomnia; 2.Improvement in quality of life; 3.Clinical efficacy or clinical cure rate.

Additional outcome(s): Secondary outcomes will include: (a) the total scores of the Insomnia Severity Index (ISI);(b) syndrome according to standards for assessing traditional Chinese medicine; and (c) adverse events, such as nausea, dizziness, vomiting, and fatigue.

Data management: First, we design an extraction form that meets the purpose of this system review, which will include the following information from the included studies: participant characteristics, interventions, outcomes, and adverse events. Two investigators (QZS and ZLL)will independently completed the data collection form for all eligible studies. The corresponding authors will be contacted to request insufficient or missing information. Disagreements will be resolved by discussion or by appealing to a third author (WZH). The data were stored in Microsoft Excel. When there are events in the reports that are unclear or do not report data, we will contact the author by phone or email to obtain complete information.

Quality assessment / Risk of bias analysis: We used the Cochrane risk assessment tool to assess the risk of bias.The methodological quality of RCTs will be independently evaluated by 2 reviewers (FZT and QZS)The following 7 items will be included: random sequence generation, allocation concealment, blinding of participants and caregivers, blinding of outcome evaluator, incomplete outcome data, selective reporting, and other bias. High, low, and unclear assessments were performed for each item. Any disagreement between the two reviewers (FZT and QZS) will be resolved by a discussion. Further disagreements were arbitrated by the third author (WZH).

Strategy of data synthesis: RevMan5.4.1 will be used for all statistical analyses. We used the random effects model to merge the data. The results of the meta-analyses are presented as forest plots. When the results are unsuitable for combination due to clinical or the methological heterogeneity, we will perform a descriptive analysis.

Subgroup analysis: If there are adequate studies and available data, we will conduct subgroup analysis for different syndrome types of insomnia to explain the heterogeneity among studies.

Sensitivity analysis: If the result shows high heterogeneity ( the 12 test is $>50 \%$ ), we will 
conduct a sensitivity analysis. We will then acquire a stable result of our study.

Language: No language to limit.

Country(ies) involved: China.

Keywords: Abdominal acupuncture; Insomnia; Systematic review; metaanalysis; protocol.

Contributions of each author:

Author 1 - Zhitao Feng - Investigation. Software. Writing - original draft. Writing review \& editing.

Email: 643628848@qq.com

Author 2 - Zhihong Wang - corresponding Methodology. Project administration. Supervision. Writing - review \& editing.

Email: 812839353@qq.com

Author 3 - Zhuangshuang Qiu - Software.

Writing - original draft.

Email: 951091309@qq.com

Author 4 - Tie Li - Methodology.

Supervision. Writing - review \& editing.

Email: 641368818@qq.com

Author 5 - Lili Zhang - Investigation.

Email: 531008689@qq.com

Author 6 - Jiajia Wang - Investigation.

Email: 583774143@qq.com

Author 7 - Dashi Ying - Project administration.

Email: 981016773@qq.com 